\title{
Temsirolimus verlängert progressionsfreies Überleben
}

Seit August 2009 ist der mTOR-Inhibitor Temsirolimus EU-weit für die Rezidivtherapie des Mantelzell-Lymphoms (MCL) zugelassen. Damit steht erstmals eine spetifisch zugelassene Option für Patienten mit rezidiviertem und refraktärem MCL zur Verfügung.

Charakteristisch für das MCL ist die durch die Translokation $t(11 ; 14)$ q(13;32) bedingte Überexpression von Cyclin D1. „Cycline sind die Motoren im Zellzyklus, sodass die Überexpression in einer starken Proliferation resultiert", erläuterte Georg Heß, Mainz. Der Erkrankungsverlauf beim MCL ist variabel: Neben indolenten ist oft auch mit sehr aggressiven Verläufen zu rechnen. Mit allogener Stammzelltransplantation, Induktions- und konsolidierender Hochdosistherapie sowie Immunchemotherapie stehen für die Primärtherapie mehrere Optionen zur Auswahl. Allerdings sind Rezidive nach zwei bis drei Jahren unvermeidlich.

Die Zulassung von Temsirolismus (Torisel ${ }^{\circledR}$ ) für die Behandlung des rezidivierten oder refraktären MCL beruht auf einer Studie mit 162 intensiv vorbehandelten Patienten (Hess G et al., 2009, J Clin Oncol 27: 3822-3829). Auf Temsirolimus in der jetzt zugelassenen Dosierung (175mg/Woche für drei Wochen, dann $75 \mathrm{mg} /$ Woche) sprachen $22 \%$ der Patienten an, während die Ansprechrate im Kontrollarm (Therapie nach Investigator's Choice) lediglich $2 \%$ betrug. Die Patienten im Kontrollarm überlebten median nur 1,9 Monate ohne Progression, die mit Temsirolimus behandelten Patienten dagegen median 4,8 Monate $(p=0,0009)$.

\section{Neuer Therapiealgorithmus und neue Perspektiven}

Nach einem von Martin Dreyling, München, entwickelten Therapiealgorithmus sollte Temsirolimus bei Patienten mit gutem Allgemeinzustand in der dritten Therapielinie zum Einsatz kommen. Bei Patienten mit schlechtem Allgemeinzustand ist der mTOR-Inhibitor bereits beim ersten Rezidiv indiziert.

Mit neuen Konzepten zum Einsatz von Temsirolimus in früheren Therapielinien und im Rahmen von Kombinationen soll das Outcome beim MCL weiter verbessert werden. In mehreren deutschen Zentren wurde die Phase-I/II-Studie BERT mit der Kombination Bendamustin/Rituximab/Temsirolimus bei Patienten im ersten bis dritten Rezidiv begonnen. Da der mTOR-Signalweg auch bei anderen Lymphomentitäten überaktiviert ist, wird Temsirolimus weiterhin bei follikulären Lymphomen und diffus-großzelligen B-Zell-Lymphomen (DLBCL) geprüft. Ein Beispiel ist die STORM-Studie, in der Temsirolimus bei Patienten mit rezidiviertem bzw. therapierefraktärem DLBCL in Kombination mit einer intensiven Immunchemotherapie (Rituximab-Dexamethason/Cisplatin/Cytarabin [R-DHAP]) eingesetzt werden soll. $K A$

Quelle: Veranstaltung der Pfizer Deutschland GmbH

\section{Nilotinib: Zulassung als CML-Erstlinientherapie} Die European Medicines Agency (EMA) erteilte die EU-weite Zulassung für Tasigna ${ }^{\circledR}$ (Nilotinib 2 × 300mg/Tag, Novartis Phar$\mathrm{ma} \mathrm{GmbH)} \mathrm{zur} \mathrm{Therapie} \mathrm{erwachsener}$ Patienten mit neu diagnostizierter Philadelphia-Chromosom positiver chronischer myeloischer Leukämie (CML) in der chronischen Phase. Die Zulassung basiert auf den 12-Monats-Daten der ENESTndStudie (Saglio G et al., 2010, N Engl J Med 362: 2251-2259). In dieser Studie hat sich eine Erstlinientherapie mit Nilotinib im Vergleich zum bisherigen Standard Imatinib in klinisch relevanten Parametern als wirksamer erwiesen. Wie die 18-Monats-Daten zeigen, waren unter Nilotinib nur zwei Patienten (0,7\%) progredient, unter Imatinib hingegen zwölf Patienten $(4,2 \%$; $p=0,006$; Larson R et al., ASCO 2010: \#CRA6501; Hochhaus A et al., EHA 2010:\#1113)

\section{Durchbruchschmerzen bei Tumorpatienten}

\section{Transmukosale Fentanyl- Applikation als Fortschritt}

Trotz Schmerzbehandlung kommt es bei etwa zwei Drittel der Tumorpatienten oft mehrmals täglich zu Durchbruchschmerzen. Diese verlangen nach einer unverzüglichen Behandlung, da die hohe Schmerzintensität die Patienten unerträglich belastet und ihre Lebensqualität mindert.

„Die ideale Durchbruchschmerz-Medikation muss daher schnell und kurz wirken und sollte nach Möglichkeit nicht invasiv sein", so FrankElsner, Aachen. Diesen Anforderungen entspricht nach Einschätzung von Hans Georg Kress, Wien/Österreich, transmukosales Fentanyl - beispielsweise in Form von Fentanyl-Buccaltabletten (Effentora ${ }^{\circledR}$ ). Durch den buccalen Applikationsweg lässt sich eine Durchbruchschmerz-Attacke entscheidend schneller behandeln, da sich die volle analgetische Wirkung bereits nach zehn Minuten entfaltet (Slatkin NE et al., 2007, J Support Oncol 5: 327-334).

Wichtig ist, in diesem Zusammenhang mit Irrtümern und Vorurteilen aufzuräumen. Die Erkenntnis, dass bei Durchbruchschmerzen das Schmerzmaximum bereits nach wenigen Minuten einsetzt und die Schmerzen in der Regel nicht länger als 30 Minuten anhalten, hat sich laut Kress noch immer nicht ausreichend durchgesetzt. Kurzwirksame Opioide sind in diesem Zeitfenster nicht wirksam. Auch die OpioidDauermedikation kann diese Schmerzspitzen nicht auffangen. Für Kress sind transmukosale Fentanyl-Applikationsformen der neue Standard in der Behandlung von Durchbruchschmerzen. $B R$

Quelle: Veranstaltung der Cephalon GmbH 